\title{
Improving VHT MU-MIMO Communications by Concatenating Long Data Streams in Consecutive Groups
}

\author{
Aitizaz Uddin Syed and Ljiljana Trajković \\ Simon Fraser University \\ Vancouver, British Columbia, Canada \\ Email: \{asyed, ljilja\}@sfu.ca
}

\begin{abstract}
Very High Throughput Multi-User Multiple Input Multiple Output (VHT MU-MIMO) is an 802.11ac communication mode that allows an Access Point (AP) to simultaneously transmit multiple data streams as Aggregated Multi-Protocol Data Units (A-MPDUs) to a group of multiple stations (STAs) over the same channel. This mode combines communication technologies that enable the 802.11ac protocol to use spectrum more efficiently compared to the previous standards. However, VHT MU-MIMO wastes an unused part of the Physical Protocol Data Unit (PPDU) interval when short and long data streams are grouped together.
\end{abstract}

In this paper, we propose a solution that improves VHT MUMIMO communications by reducing wasted portion of the PPDU duration of short data streams by concatenating longer data streams in consecutive groups. Simulations of the VHT MUMIMO communication process with and without the proposed approach indicate smaller wasted part and shorter transmission time of randomly generated STAs data streams.

Keywords-802.11ac; Multi-User-Multiple Input Multiple Output; Frame aggregation; Block acknowledgment.

\section{INTRODUCTION}

After success of 802.11n draft 2.0, 802.11ac Task Group was created in 2007 to enable WLAN to achieve Gbps throughput range under $6 \mathrm{GHz}$ frequency band. In December 2013, the task group published an updated Amendment 4 for the 802.11ac standard [1]. In order to achieve the desired throughput over limited, shared, and unlicensed spectrum, $802.11 \mathrm{ac}$ has been upgraded with many enhancements over its predecessor $802.11 \mathrm{n}$. 802.11ac operates only on $5 \mathrm{GHz}$ while $802.11 \mathrm{n}$ operates on $2.4 / 5 \mathrm{GHz}$. The $5 \mathrm{GHz}$ frequency band is introduced with new non-overlapping channels by extending the spectrum of unlicensed free band. Unlike 802.11n that permits communication over legacy $20 \mathrm{MHz}$ and $40 \mathrm{MHz}$, $802.11 \mathrm{ac}$ transmits over $20 \mathrm{MHz}, 40 \mathrm{MHz}, 80 \mathrm{MHz}, 160 \mathrm{MHz}$, and $80+80 \mathrm{MHz}$ (non-alternate channels). The $20 \mathrm{MHz}$ primary channel selection in $802.11 \mathrm{ac}$ is dynamic and the communication channel changes on a frame-to-frame basis. 802.11 ac also introduces 256 QAM constellation point with coding rate of up to $5 / 6$, which leads to a $33 \%$ increase in data transmission [2].

In 802.11n, an Acess Point (AP) could transmit up to 4 data streams to a single station (STA) simultaneously. This is called High Throughput (HT) mode. 802.11ac increases the number of data streams to 8 for a single STA and introduces a Very High Throughput Multi-User Multiple Input Multiple Output (VHT MU-MIMO) mode where an AP may simultaneously communicate with up to 4 STAs with maximum 4 data streams per STA. In this mode, STAs are assigned a Group ID and a User Position ID. Then, using the data transmitted through VHT preambles, the respective STAs group members are informed about their number of data streams, data rates, length of data streams, error correction codes, and the common channel width. To ensure that the STAs receive only their streams of data, explicit beamforming has been standardized in 802.11ac using the Null Data Packet (NDP) sounding protocol. A high throughput was achieved by introducing $802.11 \mathrm{n}$ with frame aggregation at MAC layer in form of Aggregated Multi Protocol Data Unit. (A-MPDU) and Aggregated Multi Service Data Unit (A-MSDU) followed by Block Acknowledgement (BA). 802.11ac ensures that every transmitted frame is based on frame aggregation and block acknowledgement. The block acknowledgement is based on the Reverse Direction Protocol (RDP). Hence, receiver need not have to wait for its turn to allocate Transmission Opportunity (TXOP).

In this paper, we investigate relevant technologies introduced by $802.11 \mathrm{ac}$ that contribute to successful simultaneous transmissions of multiple streams of data to multiple STAs with very high throughput. We investigate the VHT MU-MIMO communication process and discuss its inhering drawback of wasting a part of a data stream when multiple STAs data streams of unequal sizes are selected in a group for transmission [3], [4]. To address this shortcoming, we propose a solution by concatenating longer data streams in groups. In the first group, the A-MPDU size that defines the Physical Protocol Data Unit (PPDU) duration of the group is selected based on an average A-MPDU size of all data streams. The part of the long data stream that fits in this A-MPDU is transmitted. The remaining data of the data stream is transmitted later in the next group. The end result is a reduced wasted part of a short data stream. Furthermore, the transmission time is reduced significantly for more than four data streams. We compare the proposed approach with the existing standard by simulating both scenarios using up to 100 randomly generated STAs data streams. We select a single transmission throughput from the Modulation and Coding Scheme (MCS) index so that PPDU transmit time for data streams is based on the A-MPDU selection only. The 
simulations show reduction in the wasted part of short data streams and improvement in transmission time for multiple data streams.

The remaining of the paper is organized as follows. New enhancements within 802.11ac data link and physical layers that achieve VHT MU-MIMO communication are discussed in Section II. In Section III, we combine these technologies to create a VHT MU-MIMO communication model. In Section IV, we discuss the inherent drawback in the current VHT MUMIMO communication process and suggest an approach of concatenating long data streams into groups. In Section V, we simulate and compare VHT MU-MIMO communication process with and without the proposed approach and observe differences in average transmission time for various number of data streams. Simulations show that proposed approach offers improvement in transmission time when number of data streams increases.

\section{OVERVIEW OF VHT MU-MIMO TECHNOLOGIES}

In 802.11ac, an AP may transmit data up to four STAs simultaneously in down link. This mode of communication is called VHT MU-MIMO. It is made possible by combining multiple MAC and PHY technologies that continuously operate in the background.

A. Frame Aggregation, Block Acknowledgement (BA) and Reverse Direction Protocol (RDP)

Traditionally, in wireless LANs, the stream of transmitted data was permitted to occupy a channel up to one frame at a time. Hence, the ratio of data payload and the overall overheads occupying the channel were ineffective [5]. Frame aggregation was proposed in $802.11 \mathrm{n}$ that assembles multiple frames of payload along with one time overhead required to traverse the channel. Frame aggregation may be done at two levels: Aggregated Multi Service Data Unit (A-MSDU) at the Logical Link Control (LLC) sub-layer and Aggregated MultiProtocol Data Unit (A-MPDU) at the Media Access Control (MAC) sub-layer of the Data Link layer. 802.11ac only uses AMPDU. A-MPDU provides benefit of aggregating multiple MPDUs (frames) of various types and sources to the intended destination. 802.11ac A-MPDU sizes are given in Table 1.

TABLE 1. 802.11AC A-MPDU SIZES.

\begin{tabular}{cc}
\hline Serial Number & A-MPDU sizes (octets) \\
\hline 1 & 8,191 \\
2 & 16,383 \\
3 & 32,767 \\
4 & 65,535 \\
5 & 131,071 \\
6 & 262,147 \\
7 & 524,287 \\
8 & $1,048,575$ \\
\hline
\end{tabular}

If MPDU octets in VHT MU-MIMO communication are smaller than the A-MPDU size, the remaining octets are filled with empty MPDUs. BA is a frame that acknowledges all frames successfully received inside an A-MPDU. BA may be of two forms: immediate or delayed. Immediate BA is the acknowledgment request that transmitter expects soon after a Short Interframe Spacing (SIFS) of a transmitted data. Delayed $\mathrm{BA}$ is sent later by the receiver. Finally, the RDP plays an important role for immediate BA. Using RDP, the data transmitting station signals the intended recipient to send BA after an SIFS within its TXOP duration.

\section{B. VHT Sounding Protocol}

802.11ac uses VHT sounding protocol, an explicit beamfroming mechanism where a beamformer sends a NDP (Null Data Packet) to beamformee. The beamformee receives the NDP and creates a steering feedback "V" and sends it to a beamformer. It is used by beamformer to prepare steering matrix "Q". VHT Sounding protocol ensures that it successfully manages reception of feedback one by one after feedback poll for " $n$ " STAs indicated in the field, as given in Fig. 1.

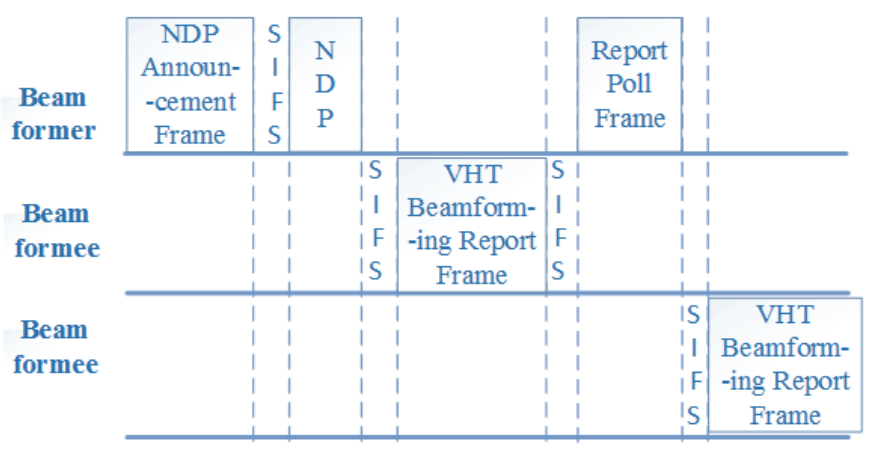

Fig. 1. A VHT Sounding process.

VHT Sounding process uses different types of frames that are signaled between beamformer and beamformee to create a steering matrix. The matrix provides steering of space-time streams in a direction of the recipient STA and nullifies the propagation to be received by other STAs, as shown in Fig. 2.

\section{Group ID Assignment}

AP assigns a Group ID and a User Position ID to STAs where it intends to simultaneously transmit data. A Group ID is assigned to up to four STAs. Each STA has a different User Position ID. An STA may be assigned multiple Group IDs. However, within the group, the User Position IDs are unique. Assignments of Group IDs are communicated to STAs via "Group ID management frame".

\section{Very High Throughput (VHT) Preamble}

802.11ac uses a mixed mode format preamble. It maintains the legacy preamble portion that resolves compatibility issues. The VHT preamble portion is enhanced to specifically accommodate multiple stream transmissions to multiple users over the same channel. It also accommodates up to 8 spacetime streams, unlike $802.11 \mathrm{n}$ HT preamble that supports only 4. VHT Preamble has two signaling fields: VHT SIG-A and VHT SIG-B. VHT SIG-A carries the collective information 
required by STAs such as intended Group ID, User Position IDs, and number of space-time stream. All training fields up to VHT-SIG-A are transmitted to all STAs, as shown in Fig. 3.

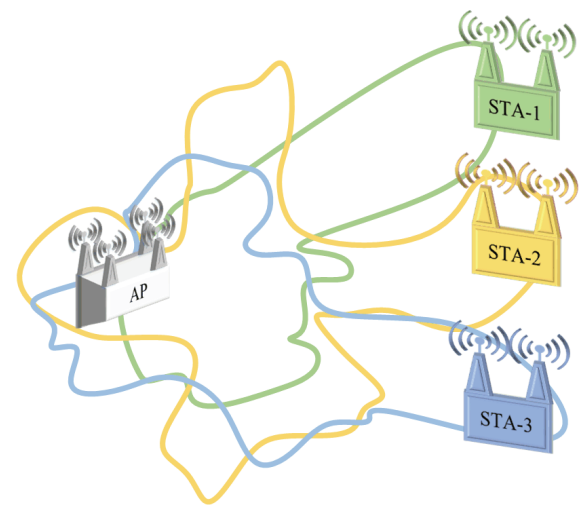

Fig. 2. Beamforming in $802.11 \mathrm{ac}$.

Following VHT SIG-A, is the VHT SIG-B signaling field. Unlike VHT SIG-A, this field is not transmitted to all STAs as one common block. Instead, multiple VHT SIG-Bs are propagated before data. Direction of VHT SIG-B propagation followed by data is a consequence of a steering matrix prepared by an AP. This signaling field has two responsibilities: 1) Identify the MCS value of a Space-Time Stream (STS) belonging to an STA. 2) Identify length of data A-MPDU in 4 octet chunks. It informs an STA to turn off its radio and save power after the STA receives data MPDUs in its A-MPDU selected from Table 1. Remaining part of an A-MPDU is filled with empty (null) MPDUs that are not required by the STA.

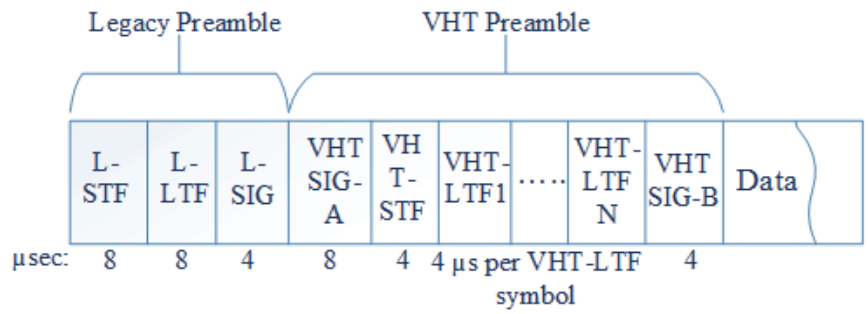

Fig. 3. 802.11ac Mixed Field Preamble.

\section{VHT MU-MIMO COMMUNICATION}

The 802.11n enhances robustness of WLAN communications via MIMO technology by introducing High Throughput (HT) mode in which an AP taking advantage of space diversity would transmit multiple data streams to single STA simultaneously on the same channel. VHT more efficiently uses spatial diversity by allowing simultaneous downlink multi-stream transmission from AP to multiple STAs. Maximum of four STAs may communicate simultaneously. The successful extraction of their own streams by STAs is the result of beamforming where space-time streams of a particular STA are directed toward the STA and streams of other STAs are nullified in its direction. When an AP decides to transmit data in downlink to multiple STAs, it starts an NDP sounding process and collects information of the STAs position. Group ID assignment phase is then performed to assign the same Group ID to STAs with a unique position ID. AP than aggregates frames of the respective STAs and selects A-MPDU sizes from Table 1 to fit the data frame octets. We assume that the STA agrees on the same MCS index for transmission. Hence, the largest A-MPDU size selected by a group member defines the PPDU duration of a group. For data streams that have fewer data MPDUs then the specified AMPDU, null MPDUs are added. After the VHT preamble, the data streams are directed based on the steering matrix. An example of VHT MU-MIMO communication process of AP with six STAs is shown in Fig. 4. Let us assume that an AP keeps tracks of all its STAs and that it has collected steering feedback from all STAs through the sounding process. The AP assigns the first Group ID to STA-1, STA-2, STA-3, and STA4. It selects an A-MPDU of 1,048,575 octets to determine PPDU duration and then transmits respective A-MPDUs. After the Block Acknowledgment procedure, the second Group ID is assigned to STA-5 and STA-6 and their A-MPDUs are transmitted. The PPDU duration is based on A-MPDU of 262,147 octets.

\section{CONCATENATING LONGER DATA STREAMS INTO GROUPS}

A portion of an A-MPDU in data streams is often wasted because after transmitting data MPDUs, an AP informs a shortstream STA to turn off its radio and save power because there are no data MPDUs to transmit. However, the channel is allocated to the group and longer data streams are transmitted in the allocated interval, as shown in Fig. 4. This unused part of a shorter data stream [3], [4] occurs because one of the data streams within the group is longer and has more data to transmit. Although the transmission time of the STAs data stream may be similar if the longer data stream has transmission throughput with a higher MCS index. Other data streams also have throughput with MCS index proportional to their length. In the worst case scenario, this may lead to longer transmission time if an STA with long data streams has large amount of data to transmit and transmission throughput with a lower MCS index.

We propose an innovative approach of concatenating long data streams into groups to reduce the unused part of a short data stream in a group. We first calculate the average of selected A-MPDUs of all data streams and select the A-MPDU size that fits the calculated average. We then reduce the size of the long data streams to the selected A-MPDU and append a Group ID assignment frame to assign the next Group ID and User Position ID. The PPDU transmission duration is selected based on the average A-MPDU. We assume that MPDU fragmentation is possible and that data are transmitted with the same MCS index. As a result, calculation of PPDU duration is only a function of A-MPDU size. The Block Acknowledgment procedure acknowledges the data streams that are terminated in the group. The next Group ID is assigned to new data streams with User Position IDs different from the one assigned to the long data steams belonging to the previous group. The size of the A-MPDU is the larger between the average of all data streams A-MPDUs and the A-MPDU that was selected by long data stream that belongs to the previous group. 


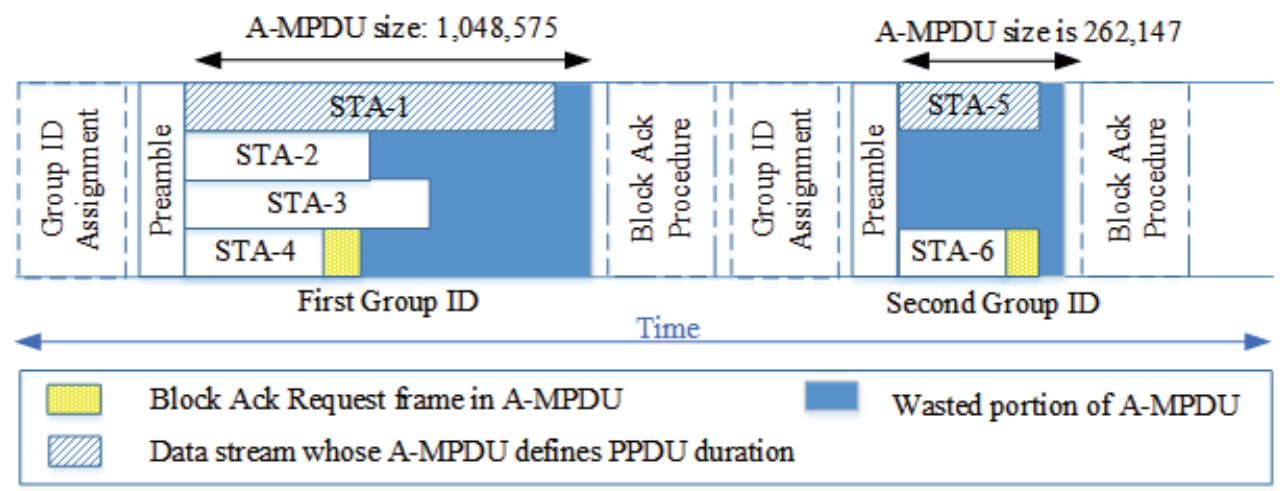

Fig. 4. VHT MU-MIMO communication process.

The proposed method considerably reduces the transmission time, as illustrated in Fig. 5. The A-MPDU of the first group of four STAs is 524,287 octets. Since data of STA-1 exceed this size, a Group ID management frame is sent to the STA that is assigning the next Group ID within the A-MPDU.
We assume the usual Block Acknowledgement and Group ID assignment process. Data from STA-1, STA-5, and STA-6 are transmitted with the A-MPDU size of 524,287 octets in the next transmitted group. The part of wasted PPDU duration is now reduced, as illustrated by comparing Fig. 4 and Fig. 5.

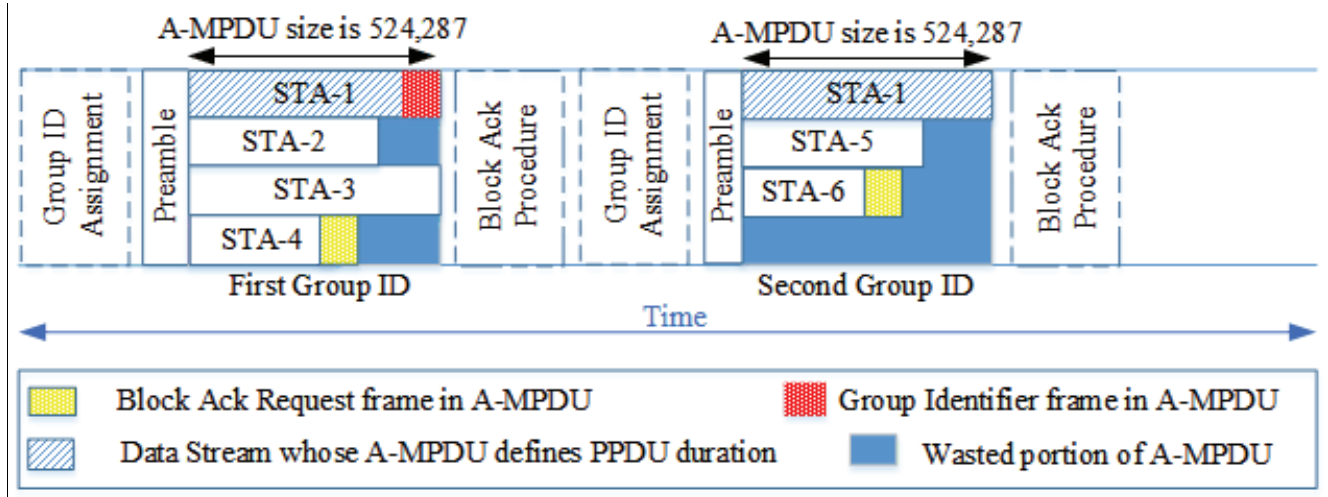

Fig. 5. Proposed approach to the VHT MU-MIMO communication process.

Taking average of A-MPDUs in a group provides significant improvement to the VHT MU-MIMO throughput. In two cases, the average of A-MPDUs is not selected as the AMPDU size for the group: 1) If a data stream, whose part was transmitted in the previous group, should complete its transmission in the next subsequent group. Hence, the AMPDU size of the group may be the largest A-MPDU size of the data stream belonging to the previous group. The reason is that the entire data stream should be acknowledged using one BA frame and its acknowledgement should not be delayed by splitting its transmission in multiple groups. 2) If at least two data streams select similar A-MPDU size, that A-MPDU size is selected as the A-MPDU size for the group. It offers two benefits: It ensures completion of at least two data streams and significant portion of other two streams. It also avoids a situation when selection of the A-MPDU as an average leads to benefit of only few data streams while wasting octets of other data streams. For example, consider a case when the A-MPDU sizes of data streams are: 8,191, 524,287, 524,287, and $1,048,575$. The A-MPDU size for the group is the average of the A-MPDUs $(1,048,575)$. With this choice, two data streams are empty halfway and the third only covers $1 / 128$ of the allocated PPDU. However, if the A-MPDU size of 524,287 is selected, three data streams consume their entire PPDU duration and the wasted portion of the fourth data stream is reduced by half. The process of selecting the A-MPDU size is shown in Fig. 6.

The waste in PPDU duration of a short data stream in a VHT MU-MIMO communication makes multiple users frame aggregation (MU-FA) scheme a viable option because it leads to better throughput [3]. However, the goal of the MU-MIMO technology in 802.11ac is to focus on service delivery by AP to small handheld STAs that communicate using limited throughput [6]. High throughput is not essential for such MUMIMO communication. The proposed solution reduces the wasted portion of PPDU durations and enhances the MUMIMO throughput of the current standard by preserving compatibility. It also enables an AP to serve more STAs in less time since additional data are packed in the data streams.

\section{Simulation RESUlts}

We simulate the proposed approach with up to 100 data streams generated randomly. The size of data streams ranges between 2,000 and 1,048,575 octets in order to select various A-MPDU sizes from Table 1. We assume that all STAs agree 
on the same MCS index for transmission. Hence, only the AMPDU size defines the PPDU duration. We consider two simulation scenarios. We first calculate transmission time of the data streams using the standard VHT MU-MIMO communication process. The data stream with the largest AMPDU size defines the PPDU duration of a group. We then simulate transmission of data streams using the proposed solution that concatenates longer data streams into groups. We use the following assumptions in order to evaluate the difference in transmission time of both processes: 1) All STAs agree on transmission throughput of $26 \mathrm{Mbps}$, which is equivalent to the VHT MCS index 3 of a single space-time stream with 16 QAM modulation of rate 1/2. 2) All STAs are receiving data with the Long Guard Interval. 3) Only Block Convolution Coding (BCC) is used. 4) The AP VHT Sounding process keeps track of all STAs positions over time. 5) Delayed Block Acknowledgment is selected for communication. 6) MPDU frames inside the A-MPDU may be fragmented. The transmission time over the channel is [1]:

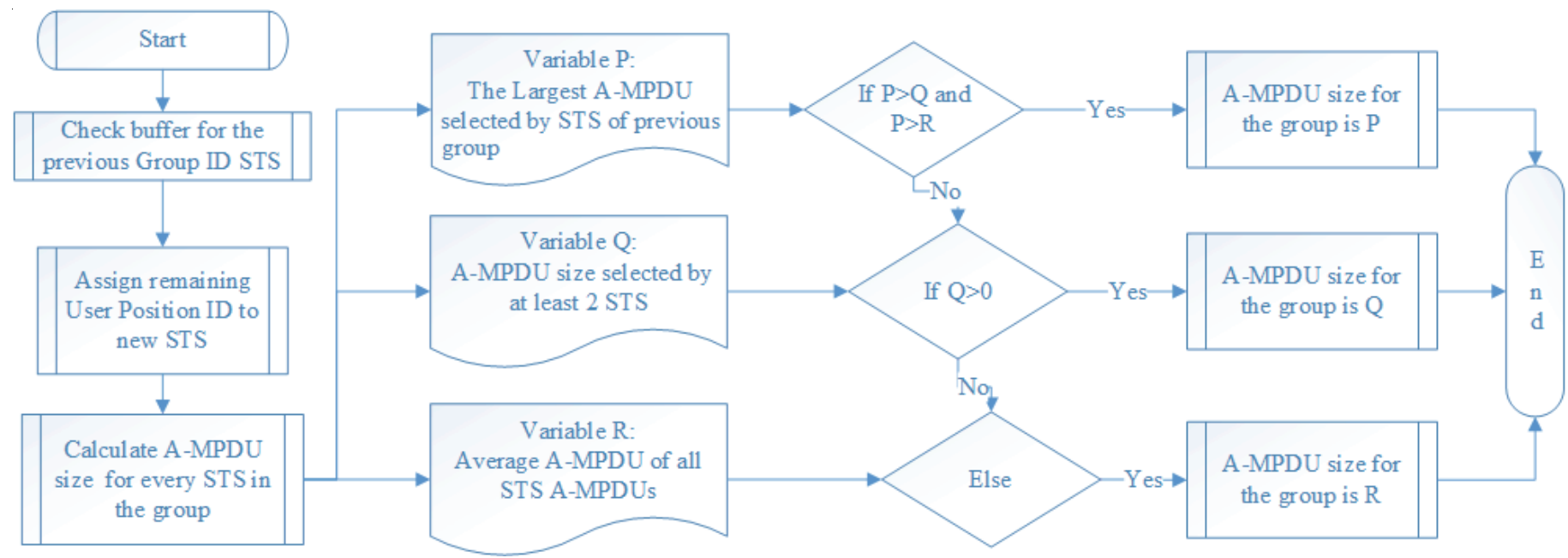

Fig. 6. Selection of A-MPDU size for a group.

$T X T I M E=T_{L S I G_{-} P R}+T_{L-S I G}+T_{V H T-S I G-A}+T_{V H T-P R}+$ $T_{V H T-S I G B}+T_{S Y M L} \times N_{S Y M}$

The preambles value is:

$T_{L S I G_{-} P R}+T_{L-S I G}+T_{V H T-S I G-A}+T_{V H T-P R}+T_{V H T-S I G B}=$ $40 \mu \mathrm{s}$.

The $\mathrm{N}_{\mathrm{SYM}}$ of the data A-MPDU is:

$N_{S Y M}=m_{S T B C} \times\left\lceil\left(8 \times A P E P_{-} L E N G T H+N_{\text {service }}+\right.\right.$ $\left.\left.N_{\text {tail }} \times N_{E S}\right) /\left(m_{S T B C} \times N_{D B P S}\right)\right]$,

where $T_{S Y M L}=4, m_{S T B C}=1, N_{\text {service }}=16, N_{\text {tail }}=8, N_{E S}=1$, $N_{D B P S}=104$, and APEP_LENGTH $=$ A-MPDU size is selected from Table 1 for STAs within a group.

In addition to data transmission intervals, Block Acknowledgment and Group ID assignment intervals are also necessary for the VHT communication process, as shown in Fig. 4 and Fig. 5. The intervals comprise of transmission of multiple management and control frames. These frames increase with the increased number of data streams. After control frame, management frame, and data stream, there is a transmission pause called Short Inter Frame Spacing. The duration of frames is calculated at transmission throughput of $26 \mathrm{Mbps}$, which is equivalent to the VHT MCS index 3 [7]. Duration of frames is shown in Table 2.

We calculate transmission time of data streams using (1) and (2) and add the time required by the control/management frames and SIFS. Simulations using randomly generated data streams are shown in Fig. 7. The proposed approach significantly reduces the transmission time compared to the standard VHT MU-MIMO communication process. In simulations of 100 data streams, 4 data streams are assigned to a group by the current 802.11 ac standard thus utilizing 25 Group IDs. In the proposed approach, the requirement for Group IDs increases to 27-30 because some data streams belong to two groups. The increase of Group IDs may increase the number of Group ID management frames. Nevertheless, the number of frames is comparable to the standard VHT MUMIMO process because every long data stream is assigned the next Group ID within an A-MPDU. Furthermore, the number of BA and BAR frames is slightly smaller because additional groups provide an opportunity to transmit additional BAR frames in the A-MPDUs. Hence, the proposed approach does not create additional overhead in time and no extra TXOP needs to be allocated to control and management frames.

TABLE 2. DURATION OF CONTROL FRAMES, MANAGEMENT FRAMES, AND INTERFRAME SPACING

\begin{tabular}{lcl}
\hline \multicolumn{1}{c}{ Description } & $\begin{array}{c}\text { Duration } \\
(\mu \mathrm{s})\end{array}$ & \multicolumn{1}{c}{ Comment } \\
\hline $\begin{array}{l}\text { Block Acknowledgement; } \\
\text { Block Acknowledgement Request }\end{array}$ & 54 & $\begin{array}{l}\text { Transmission time } \\
\text { includes preamble }\end{array}$ \\
Group ID assignment frame & 60 & $\begin{array}{l}\text { Transmission time } \\
\text { includes preamble } \\
\text { Short Interframe Spacing }\end{array}$ \\
\hline
\end{tabular}


Performance results are verified by repeating simulations 10 times for 100 distinct data streams. The proposed approach significantly reduces the number of wasted octets of short data streams, as shown in Fig. 8. The transmission time is reduced by at least $0.5 \mathrm{~s}$, as shown in Fig. 9. The proposed approach does not introduce additional overhead of control and management frames, as shown in Fig. 10.

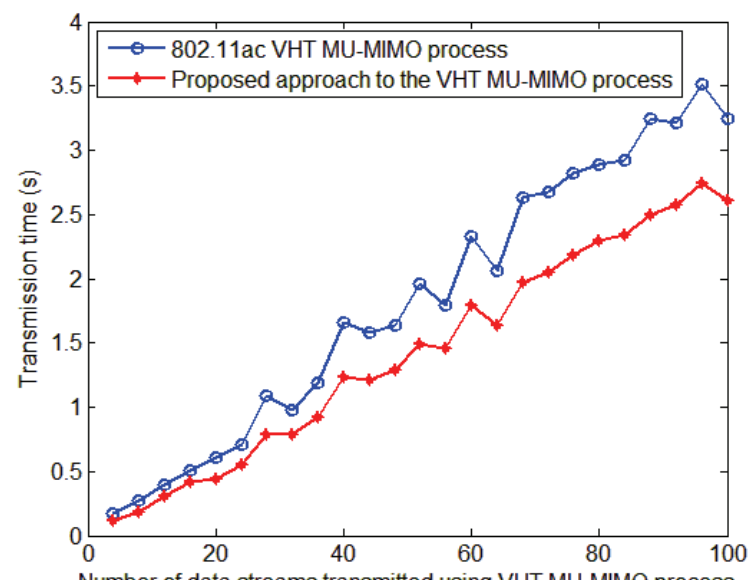

Fig. 7. Comparison of the proposed approach and the VHT MU-MIMO process.

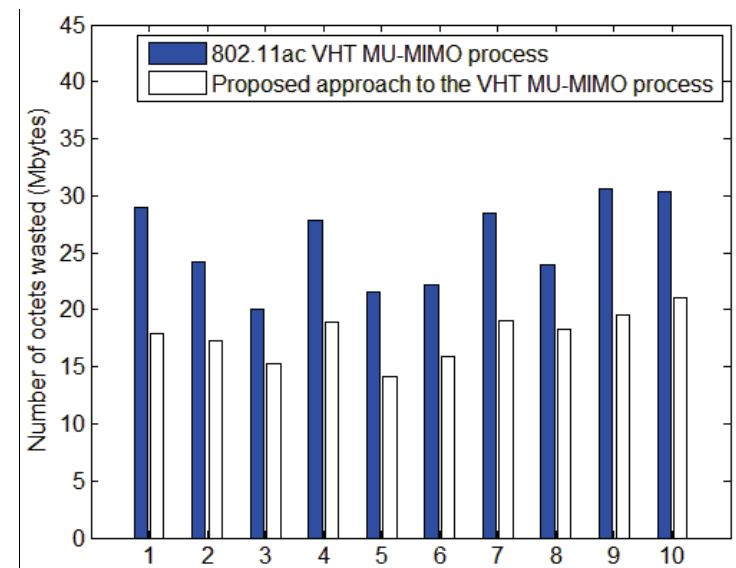

Fig. 8. Performance comparison of 10 simulations: Number of wasted octets.

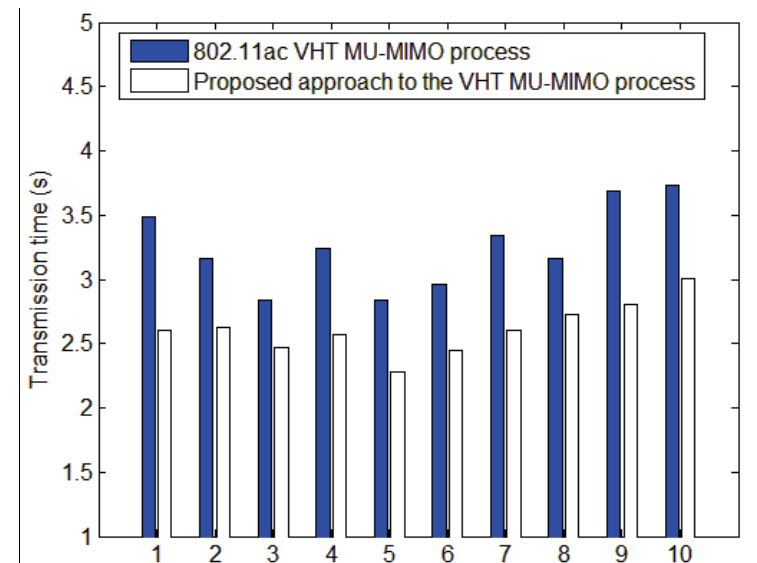

Fig. 9. Performance comparison of 10 simulations: Transmission time.

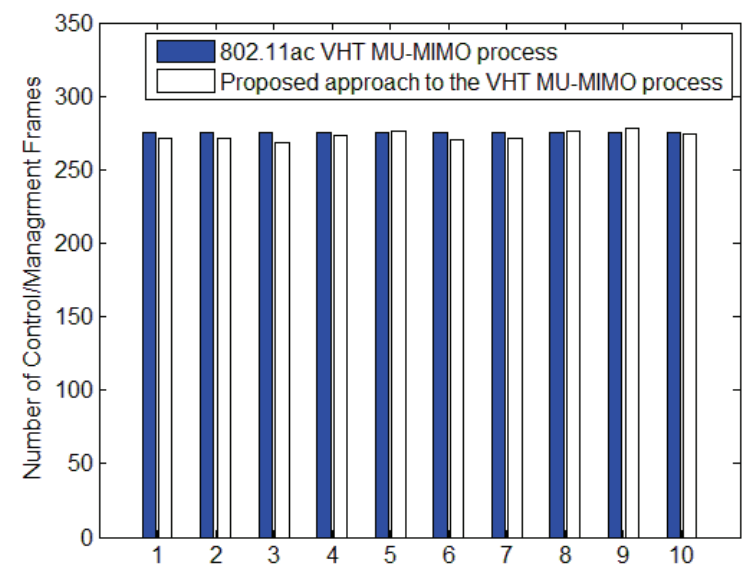

Fig. 10. Performance comparison of 10 simulations: Control /Management frames.

\section{CONCLUSION}

In this paper, we explore the VHT MU-MIMO communication mode with its supporting technologies. We propose a solution to reduce wasting a portion of an A-MPDU of a short data stream in a group of un-equal streams by concatenating longer data streams in consecutive groups. In the proposed solution, we consider selection of PPDU duration of a group as function of A-MPDU size and divide the A-MPDU of long data stream to the average of the entire group while the next consecutive Group ID is assigned within the A-MPDU. After the Block Acknowledgement procedure and Group ID assignment to new STAs, remaining data of the long data streams are transmitted together with the new data streams. Simulation results show significant improvement in transmission time and efficient space-time utilization of channel with no additional overhead of control and management frames.

\section{REFERENCES}

[1] Part 11: Wireless LAN Medium Access Control (MAC) and Physical Layer (PHY) Specifications. Amendment 4: Enhancements for Very High Throughput for Operation in Bands below $6 \mathrm{GHz}$, IEEE Standard 802.11ac, 2013.

[2] M. Gast, 802.11ac, A Survival Guide. Sebastopol, CA, USA: O’Reilly, 2013.

[3] C. Jiyoung, J. Hu, B. C. Jung, and D. K. Sung. "Performance Comparison of Downlink User Multiplexing Schemes in IEEE 802.11ac: Multi-User MIMO vs. Frame Aggregation," in Proc. Wireless Communications and Networking Conference (WCNC), Paris, France, Apr. 2012, pp. 1514-1519.

[4] C. Chung, K. Chung, B. Kang, and J. Kim, "A-MPDU using Fragmented MPDUs for IEEE 802.11ac MU-MIMO WLANs," in Proc. TENCON 2013 IEEE Region 10 Conference, Xi'an, China, Oct. 2013, pp. 1-4.

[5] D. Skordoulis, Q. Ni, H. Chen, A. P. Stephens, C. Liu, and A. Jamalipour, "IEEE 802.11n MAC frame aggregation mechanisms for next-generation high-throughput WLANs," IEEE Wireless Communications, vol. 15, no. 1, pp. 40-47, Feb. 2008.

[6] (October 9, 2014) Cisco. "802.11ac: The Fifth Generation of Wi-Fi Technical White Paper." [Online]. Available: http://www.cisco.com/ en/US/prod/collateral/wireless/ps5678/ps11983/white_paper_c11713103.html.

[7] E. H. Ong, J. Kneckt, O. Alanen, Z. Chang, T. Huovinen, and T. Nihtila, "IEEE 802.11ac: Enhancements for very-high throughput WLANs," in Proc. IEEE Personal Indoor and Mobile Radio Communications (PIMRC), Toronto, ON, Canada, Sept. 2011. pp. 849-853. 